\title{
ALGUNAS CONSIDERACIONES SOBRE LA NOCIÓN DE ANALOGIA EN TOMÁS DE AQUINO EN EL CONTEXTO DEL PROBLEMA DE LOS NOMBRES DIVINOS
}

\author{
Some Considerations about the Notion of Analogy in \\ Thomas Aquinas in the Context of the Problem of the Divine Names
}

\author{
Dra. Rosa Elvira Vargas \\ Universidad Femenina Sagrado Corazón: rosavargasd@unife.edu.pe \\ CÓDIGO ORCID: 0000-0002-9293-1595
}

\begin{abstract}
RESUMEN
El presente trabajo ofrece una aproximación a la teoría tomista de la analogía de los nombres divinos a partir de la consideración de la noción general de analogía que subscribe Tomás, la cual tiene su origen en las discusiones en torno a la equivocación en los tratados de lógica. Se muestra de qué manera la predicación análoga de Dios y de la creatura es un caso único, no enteramente equiparable con los casos tradicionales de predicación análoga. Las diversas caracterizaciones de la analogía de los nombres divinos que ofrece Tomás responderían, más que a una evolución de su pensamiento sobre la analogía, a un intento de caracterizar dicha diferencia.
\end{abstract}

\section{Palabras clave:}

Tomás de Aquino, analogía, equivocación, nombres divinos.

\begin{abstract}
The paper offers an approximation to the Thomistic theory of the analogy of the divine names from a consideration of the general notion of analogy to which Aquinas subscribes and which has its origin on the discussions on equivocation found in logical treatises. It shows the extent to which the analogous predication of God and of creature is a unique case, not entirely equal to the traditional cases of analogous predication. The diverse ways in which Aquinas characterizes the analogy of the divine names would respond, not so much to an evolution of his thoughts on analogy, but to an attempt to characterize such difference.
\end{abstract}

\section{Keywords:}

Thomas Aquinas, analogy, equivocation, divine names. 


\section{INTRODUCCIÓN}

Uno de los problemas al que nos enfrentamos al momento de abordar la teoría tomista de la analogía de los nombres divinos es el de determinar cómo debe entenderse se da la predicación análoga de Dios y de la creatura, esto es, bajo qué tipo de analogía debemos entender es posible predicar un mismo nombre de Dios y de la creatura. En el ámbito de las discusiones sobre la analogía se suele distinguir entre la analogía de atribución y la de proporcionalidad; sin embargo, tal distinción tiene como fuente principal a Cayetano y no se correspondería en estricto sentido con el tratamiento que el propio Tomás hace de los tipos de analogía (Porro, 2018). Ahora bien, la tipología tomista tampoco nos ofrece mucha ayuda. Por lo general se distinguen dos tipos, pero en ocasiones se distinguen tres tipos de analogía ${ }^{1}$ - De otro lado, independientemente de la tipología utilizada, Tomás no es consistente al contrastar la analogía de los nombres divinos con los casos emblemáticos de analogía. En algunos casos la analogía de los nombres divinos se distingue claramente de la analogía de los términos 'ente' y 'sano', pero en otros casos más bien se la equipara con uno u otro caso ${ }^{2}$. Tomás parece cambiar de parecer, corrigiéndose y en ocasiones volviendo sobre sus pasos, lo que ha sido tomado como evidencia de una evolución en el pensamiento tomista con respecto a la relación analógica entre Dios y la creatura (Lonfat, 2004; Ashworth, 2008). Frente a estas dificultades, considero que una aproximación a la teoría tomista de la analogía de los nombres divinos que parte, no de una consideración de tipos de analogía, sino de la noción general de analogía subyacente a las diversas tipologías ofrecidas, nos conduciría a una comprensión más amplia y profunda del tratamiento del problema por parte de Tomás. Tal aproximación se justifica al observar que a lo largo de su obra Tomás es consistente en señalar que la predicación de los nombres divinos no es ni unívoca, ni equívoca, sino análoga.

El trabajo se divide en tres partes. En la primera sección, reviso las fuentes que enmarcan el tratamiento de la teoría de la analogía hacia mediados del siglo XIII. En la segunda sección, con el objetivo de caracterizar la noción general de analogía que maneja Tomás, presento la postura tomista en torno a algunas cuestiones que dominan la discusión sobre la analogía durante la época. Finalmente, en la tercera sección, a la luz de lo desarrollado en las secciones precedentes, considero el tratamiento que Tomás hace de la analogía de los nombres divinos.

\section{Las fuentes de la teoría de la analogía en el siglo XIII}

Antes de pasar a la revisión de las fuentes, conviene recalcar dos puntos importantes sobre el uso de la palabra 'analogía' en el siglo XIII. Primero, la noción de analogía hace referencia a un modo de predicación, según el cual una misma palabra o término es predicado de dos o más sujetos con significados diversos pero similares a la vez. Se trata de una noción lógica o semántica que más adelante es trasladada al ámbito ontológico. En consecuencia, no debe

\footnotetext{
1 Por ejemplo, en el comentario a las Sentencias, Tomás inicialmente distingue tres tipos (In I Sent., d. 19, q. 5, a. 2, ad 1), pero más adelante distingue dos (In I Sent., d. 35, q. 1, a. 4). En obras posteriores, lo predominante son dos tipos (cf. De ver., q. 2, a. 11; ST I, q. 13, a. 5).

2 Cf. ST I, q. 13, a. 5 y SCG I, c. 34.
} 
sorprender que las discusiones sobre la analogía en el siglo XIII tengan como marco general las discusiones lógico semánticas en torno a la equivocación, esto es, a los términos que tienen más de un significado (Ashworth, 1992, p. 97). Segundo, aun cuando la palabra 'analogía' suele asociarse a la afirmación de Aristóteles sobre los múltiples significados de 'ser' y 'ente', el hecho es que Aristóteles nunca hace uso del término griego 'analogía' (ava $\lambda$ oyıa) para caracterizar la multitud de significados de los términos 'ser' y 'ente'. La palabra griega avaגoyıa (traducida algunas veces por 'analogía' otras por 'proporción' o 'proporcionalidad') se refiere a una comparación entre dos proporciones. Es sólo a partir de 1220 , por influencia de la filosofía árabe, que el término 'analogía' adquiere el significado relevante para nuestra discusión.

Paso a continuación a considerar las tres fuentes principales que enmarcan las discusiones en torno a la analogía en el siglo XIII.

\section{a. El comentario de Boecio a las Categorías de Aristóteles}

Al inicio de las Categorías (1a1-9), distinguiendo entre términos unívocos y equívocos, Aristóteles señala que los términos unívocos comparten una misma palabra y un mismo concepto; mientras que los términos equívocos comparten una misma palabra, pero el concepto es diferente. En su comentario al texto de Aristóteles, Boecio distingue a su vez dos clases de términos equívocos: equívocos por accidente (aequivoca a casu) y equívocos por acuerdo (aequivoca a consilio). Estos últimos, cuya característica distintiva es la existencia de una relación entre los diversos usos del término, son subdivididos en cuatro clases según el tipo de relación existente: (i) similitud; (ii) proporción (proportio en latín y $\alpha v \alpha \lambda o y ı \alpha$ en griego); (iii) ab uno, como es el caso de 'medicinal'; y (iv) ad unum, como es el caso de 'sano's. En la literatura posterior a Boecio, las dos últimas subdivisiones tienden a agruparse bajo una misma denominación, generalmente ut unum (Ashworth, 1992, pp. 101-102). En el siglo XIII, sin embargo, por influencia de la filosofía árabe, esta denominación común cambia a analogia, aunque ya no en el sentido original griego, sino en relación con la noción de prioridad y posterioridad (secundum prius et posterius) (Ashworth, 1996, p. 237). Sobre este último punto regresaremos más adelante.

\section{b. Las discusiones en torno a la equivocación en las Refutaciones sofísticas de Aristóteles}

En Refutaciones sofísticas 166a15-20, Aristóteles distingue tres modos de equivocación: (i) cuando un mismo término significa más de una cosa, (ii) cuando un mismo término se usa de manera equívoca por convención, y (iii) cuando dos o más términos significan en conjunto más de una cosa, pero por separado sólo una cosa. Al respecto, Pedro Hispano, en su Tractatus, explica que el segundo modo de equivocación al que hace referencia Aristóteles involucra el caso de diversas cosas significadas por un mismo nombre según prioridad y posterioridad, como cuando 'sano' se dice de una persona y de la orina (De Rijk, 1972, p. 100) ${ }^{4}$. Más adelante, distinguiendo diversos modos de unidad,

3 Para un tratamiento más profundo de las divisiones de Boecio, véase Ashworth (1996, pp. 235-236).

4 Escrito entre 1230 and 1245, el Tractatus es señalado como el libro de lógica más influyente del medioevo; fue usado como libro de texto hasta entrado el siglo XVII (De Rijk, 1972, pp. XcV-cx). 
Pedro identifica un modo de unidad de 'proporción' que es aquella de lo que se dice según prioridad y posterioridad. Como ejemplos de este modo de unidad señala: 'ente', dicho de todo lo que es; 'sano', dicho de todas las cosas sanas; y 'bueno', dicho de todas las cosas buenas (De Rijk, 1972, p. 177).

Con respecto al término 'ente', en otro pasaje del Tractatus, esta vez en el contexto de la distinción entre la predicación univoca y la equívoca, Pedro explica que 'ente' no se predica unívocamente sino equívocamente porque no se predica de varios sujetos según un mismo concepto, como es el caso de la predicación unívoca, sino que se predica de varios de acuerdo con diversos conceptos. Así, 'ente' se dice de la sustancia según el concepto de ente por sí y se dice de los accidentes según el concepto de ente en otro (De Rijk, 1972, p. 25). La referencia a la distinción aristotélica entre términos unívocos y equívocos en las Categorías es clara. En el caso de la univocidad tenemos un mismo nombre que se predica de muchos según un mismo concepto; en el caso de la equivocidad tenemos un mismo nombre que se predica de muchos según diversos conceptos. Ahora bien, según lo desarrollado por Pedro Hispano en los pasajes antes señalados, estos conceptos no serían totalmente diversos porque habría entre ellos una cierta unidad de proporción según prioridad y posterioridad.

\section{c. La Metafísica de Aristóteles y los filósofos árabes}

La recuperación de la Metafísica de Aristóteles en el siglo XIII y con ella el trabajo de los filósofos árabes añade otros matices a la discusión (Ashworth, 1992, pp. 107-109; 2008, pp. 28-31). En
Metafísica 4.3 (1003a33-35), Aristóteles afirma que 'ente' se dice tanto de la sustancia como de los accidentes, pero no de manera equívoca. Esto contradice directamente lo señalado por Porfirio en la Isagoge, a saber, que 'ente' se dice equívocamente. Para disolver la aparente contradicción, se apela a los modos de equivocación que Aristóteles distingue en las Refutaciones sofísticas. Así, el término 'ente' no es equívoco según el primer modo de equivocación, pero sí lo es según el segundo modo, esto es, por convención.

El trabajo de los filósofos árabes termina de dar forma al marco conceptual de referencia para el tratamiento de la analogía en el siglo XIII. De entre ellos, destacan Averroes y Al-Ghazali. En su comentario mayor a la Metafísica (1003a33-1003b10), Averroes explica que Aristóteles clasifica los términos (i) 'sano' como un caso de relación a un fin, (ii) 'medicinal' como un caso de relación a un agente y (iii) 'ente' como un caso de relación a un sujeto. Con el fin de acomodar esta clasificación a la proporcionada por Boecio, las dos últimas subdivisiones de equívocos por convención de Boecio se expanden a tres (Ashworth, 1996, p. 236). Otra fuente importante es Al-Ghazali, quien en su Lógica hace referencia a ciertos términos ambiguos, de convergencia o conveniencia (la palabra latina es convenientia), entre ellos 'ente', que se ubican entre los términos unívocos y equívocos, y que se dicen de diversas cosas según un orden de prioridad y posterioridad (Lohr, 1965, p. 246). El uso del término convenientia en la traducción latina es interesante. Alberto Magno, en su comentario a las Categorías, indica que 'analoga' es lo que los árabes llaman 'convenientia' (Ashworth, 1992, p. 108); de otro lado, Tomás de Aquino, 
especialmente en sus primeros escritos, adopta esta terminología, identificando conveniencia y analogía 5 .

Lo señalado hasta el momento nos ofrece un panorama general de las diversas fuentes que configuran la discusión sobre la analogía en el siglo XIII. Algunos datos resaltan. En primer lugar, la analogía era entendida inicialmente como un caso de equivocidad, pero más adelante pasa a ser entendida como un caso intermedio entre univocidad y equivocidad. Segundo, en la predicación análoga, un mismo nombre es predicado de diversos sujetos bajo conceptos diversos pero relacionados entre sí a razón de cierta proporción o conveniencia de prioridad y posterioridad. Tercero, y último, la proporción o conveniencia que da lugar a la posibilidad de una predicación análoga puede manifestarse de diversas maneras.

En la siguiente sección, abordo la postura tomista en torno a una de las cuestiones más debatidas sobre la analogía entre sus contemporáneos, a saber, cómo debe entender la unidad y diversidad de conceptos en el caso de predicación análoga; o expresado de otra manera, cómo debe entenderse la convergencia o conveniencia de los términos análogos. La respuesta de Tomás a esta cuestión nos ayudará a determinar con mayor claridad la noción general de analogía bajo la cual se enmarca su tratamiento del problema de la predicación análoga entre Dios y la creatura.

\section{Sobre la unidad y diversidad de conceptos en la predicación análoga}

En el ámbito de las discusiones lógico-semánticas sobre la analogía, la cuestión de la unidad y diversidad de conceptos se plantea en términos del número de conceptos involucrados en la predicación análoga. La respuesta de Tomás varía (Ashworth, 1992, p. 124). En Los principios de la naturaleza (c. 6, n. 46), los conceptos son varios, mientras que, en el comentario a las Sentencias, se trataría de un solo concepto que es usado de diversas maneras (In I Sent., d. 19, q. 5, a. 2 ad 1). En la Suma teológica se señala que no se trata ni de uno ni de varios conceptos (ST I, q. 13, a. 5). En el comentario a la Metafísica, Tomás parece haber llegado a una postura más amplia y definida; se trata de conceptos que son parcialmente uno, parcialmente diversos:

Pero debe entenderse que algo se predica de varios de diversas maneras. A veces, se predica según un concepto completamente igual, y entonces se dice que es predicado unívocamente, como 'animal' se predica del caballo y del buey. Otras veces, se predica según conceptos que son completamente diversos, y entonces se dice que se predica equívocamente, como 'perro' se predica de la estrella y del animal. En cambio, otras veces, se predica según conceptos que son en parte diversos y en parte uno; son diversos en tanto implican diferentes relaciones y son uno en tanto estas diferentes relaciones están referidas a una misma cosa. Y esto es lo que se llama predicación análoga, es decir, proporcional (In IV Meta., lect. 1) .

Este pasaje no indica exactamente qué es aquella misma cosa que unifica los

5 En De ver., q. 2, a. 11, por ejemplo, relaciona las nociones analogía, proporción y conveniencia. Cf., también, In I Sent., d. 35, q. 1, a. 4.

6 Las traducciones de las citas corresponden a la autora de este artículo. 
conceptos al ser el foco de referencia de las diversas relaciones (habitudines) bajo las que se diferencian los conceptos. Sin embargo, en otros pasajes, Tomás explica que lo que unifica los conceptos en los casos de predicación análoga es que el concepto bajo el cual el término se predica primariamente está contenido en el concepto bajo el cual el término se predica secundariamente. Así, por ejemplo, el concepto de salud bajo el cual el término 'sano' se predica primariamente del animal está contenido en el concepto bajo el cual el mismo término se predica de la medicina ${ }^{7}$.

Resta una cuestión más por resolver en relación con la unidad y diversidad de los conceptos bajo los cuales se predica un mismo término de varios sujetos, a saber, la manera en que el concepto correspondiente a la predicación primaria se encuentra contenido en el concepto o conceptos correspondientes a las predicaciones secundarias o posteriores. Al respecto, el siguiente pasaje de De veritate nos ofrece una posible respuesta:

Lo que se dice de modo simple es algunas veces entendido de aquello que se dice con posterioridad debido a algo adjunto. Como cuando 'ente' [al adjuntarse] 'en otro' se entiende de los accidentes. De manera similar, 'vida', en razón de algo adjunto, a saber, 'libro', se entiende de la vida creada, la cual se dice 'vida' con posterioridad (De ver., q. 7, a. 5, ad 3).

Según lo expuesto, entonces, en la predicación análoga se puede hablar de cierta unidad y diversidad de conceptos, esto es, de cierta convergencia o conveniencia (convenientia) porque los conceptos secundarios o posteriores resultan de la adición de alguna especificación al concepto original o primario del término. Esto nos permite entender con más claridad el pasaje del comentario a la Metafísica citado previamente, donde se menciona que la unidad de los conceptos se debe a que las diversas relaciones (habitudines) que los distinguen tienen un único foco de referencia. Tal foco de referencia se encontraría contenido en el concepto primario a partir del cual se forma, por adición de alguna calificación, el o los conceptos posteriores. De esta manera se explica no sólo la unidad sino la diversidad de los conceptos en la predicación análoga.

A continuación, en lo que será la última sección del trabajo, paso a considerar la respuesta de Tomás al problema de los nombres divinos, esto es, a la posibilidad de predicar un mismo nombre de Dios y de la creatura. A lo largo de su obra, Tomás mantienen una postura consistente al respecto: aquello que se predica de Dios y de la creatura, no se predica de modo unívoco, ni equívoco, sino análogo.

\section{La analogía de los nombres divinos}

El problema de los nombres divinos se puede resumir con la siguiente pregunta: ¿podemos predicar algo positivo de Dios? Algunos predicados, aunque parecen afirmar algo positivo de Dios, en realidad ocultan una negación; decimos, por ejemplo, que Dios es infinito e incorpóreo, pero con ello queremos decir que Dios no es finito ni corpóreo. El caso de predicados como 'bueno' o 'sabio' es distinto; al decir de Dios que es sabio o que es bueno, predicamos efectivamente

7 Cf. ST I, q. 13, a. 6; SCG I, c. 32. 
algo positivo de Dios. Pero, he aquí el problema: nuestros conceptos de bueno y sabio tienen por objeto la bondad y sabiduría creada. Tiene pleno sentido predicarlos de Pedro o de María, pero no de Dios pues su bondad y sabiduría sobrepasan la bondad y sabiduría creada. Podríamos intentar superar esta dificultad dejando de lado los términos concretos ('bueno' y 'sabio') y haciendo uso de los correspondientes términos abstractos ('sabiduría' y 'bondad)'; sin embargo, nos seguimos enfrentando con mismo problema. El hecho es que sólo podemos concebir la sabiduría y bondad creada; la bondad y sabiduría divina trascienden nuestras capacidades intelectuales. Sin importar qué modo de significación usemos, afirma Tomás, nuestros términos se quedan cortos cuando los predicamos de Dios porque nuestros modos de significación siguen a nuestros modos de intelección ${ }^{8}$. La solución tomista a este problema es que debemos trascender el modo de significación de nuestros términos cuando los predicamos de Dios; la manera de hacerlo es a través de la analogía9 . La predicación análoga permite trascender la imperfección de los modos de significación de nuestros términos, imperfección derivada de la imperfección de nuestro modo de intelección con respecto a la esencia divina.

Ahora bien, el problema de los nombres divinos, acabamos de ver, reside en los conceptos bajo los cuales predicamos ciertas perfecciones de Dios. No podemos predicar un mismo término de Dios y de la creatura bajo el mismo concepto; si así lo hiciéramos, el término se predicaría de modo unívoco de ambos, lo cual es imposible por las razones antes expuestas. Una alternativa es predicar el mismo término, pero bajo conceptos distintos; con lo cual tendríamos una predicación equívoca. La dificultad de esta alternativa radica en que, de ser así, no podríamos predicar nada de Dios porque, como ya hemos señalado, nuestro intelecto no puede concebir un concepto tal que pueda abarcar las perfecciones divinas. Otra alternativa es la predicación análoga, según la cual predicamos un mismo término bajo conceptos que guardan cierta convergencia o conveniencia, esto es, cierta unidad y diversidad. Es cierto que no podemos concebir las perfecciones divinas, pero sí podemos aproximarnos a ellas a través de las perfecciones creadas a las que sí tenemos acceso, y esto gracias a la causalidad ejemplar y eficiente entre creador y creatura. Todo agente produce algo similar a sí; de manera que Dios, al producir las creaturas, imprime en ellas algo de sus perfecciones (Porro, 2018, p. $75)^{10}$. Al respecto, escribe Tomás:

Cualquier término que se dice de Dios y de las creaturas, se dice en cuanto hay cierto orden que guardan las creaturas con Dios como principio y causa en la que preexisten de modo excelente todas las perfecciones de las cosas. Este modo de comunidad se encuentra entre la pura equivocidad y la simple univocidad, pues en los nombres dichos por analogía no hay un concepto único, como sucede

8 Cf. In I Sent., d. 4, q. 1, a. 1; ST I, q. 13, a. 1, ad 2; De pot., q. 8, a. 2, ad 7. Sobre la afirmación de que nuestros modos de significación se siguen de nuestros modos de intelección, cf. De pot., q. 7, a. 2, ad 7; SCG I, c. 30; ST I, q. 45, a. 2, ad 2; De pot., q. 7, a. 5, ad 2; In VII Meta., lect. 1, n. 1253-54.

9 Cf. In I Sent., d. 22, q. 1, a. 2, ad 1; De pot., q. 7, a. 5, ad 2; SCG I, c. 30. Sobre este rol de la analogía en relación con los modos de significación de nuestros términos, véase Jordan (1980, p. 415); Buersmeyer (1987, p. 85); Rosier (1995, p. 155)

10 Cf. De pot., q. 7, a. 7; SCG I, c. 34; ST I, q. 13, a. 5. 
con los unívocos, ni conceptos totalmente diversos, como sucede con los equívocos, sino que el nombre que se aplica análogamente a muchos significa diversas proporciones respecto de una misma cosa; como 'sano', dicho de la orina, significa el signo de la salud, y dicho de la medicina significa la causa misma de la salud (ST I, q. 13, a. 5).

Entonces, según lo manifestado, la relación de causalidad formal y eficiente entre Dios y las creaturas garantiza que podamos trascender la imperfección de nuestros conceptos, de tal manera que podamos predicar de la creatura y de Dios perfecciones, como la bondad y la sabiduría, sin caer en la univocidad ni en la equivocidad porque los conceptos bajo los cuales se predican tales perfecciones de la creatura y de Dios no son enteramente uno ni enteramente diversos. A este tipo de predicación se le denomina 'analogía'.

En la sección anterior, señalamos que, en la predicación analógica, la unidad y diversidad de los conceptos proviene del hecho de que el concepto primario está contenido en los conceptos secundarios. Explicamos, también, que esto es así porque los conceptos secundarios o posteriores resultan de alguna calificación al concepto original o primario. Así, dijimos que 'ente' se predica del accidente añadiendo al concepto primario de ente que se predica de la sustancia el calificativo de 'en otro'. Ahora bien, la calificación al concepto primario se puede dar no sólo por adición, sino también por remoción. Este último sería el caso de los nombres divinos. Así, por ejemplo, cuando predicamos 'bueno' de Dios, removemos la noción de composición implícita en el concepto de bueno bajo el cual predicamos 'bueno' de la creatura, a la vez que retenemos la noción de subsistencia ${ }^{11}$. La causalidad formal y eficiente entre Dios y las creaturas permite que podamos realizar este tipo de calificación, en cuanto, como indica Tomás, "hay cierto orden que guardan las creaturas con Dios como principio y causa en la que preexisten de modo excelente todas las perfecciones de las cosas" (ST I, q. 13, a. 5). No debemos suponer, sin embargo, que el concepto resultante logra abarcar la perfección divina:

Dado que Dios es simple y subsistente, le atribuimos nombres abstractos para significar su simplicidad y nombres concretos para significar su subsistencia y perfección; aunque, ningún nombre es suficiente para significar su modo ser, así como en esta vida nuestro intelecto no lo conoce como es (ST I, q. 13, a. 1 , ad 2).

Aquí encontramos la diferencia central entre la predicación análoga de términos como 'bueno' y 'sabio' de Dios y de la creatura, y la predicación análoga de términos como 'ente' y 'sano' de las creaturas.

La trascendencia divina hace del caso de los nombres divinos un caso único, no enteramente equiparable con los casos tradicionales de términos análogos. Sin importar el término que usemos, no podemos nombrar perfectamente a Dios; la predicación de Dios siempre es imperfecta. La relación de causalidad eficiente y ejemplar que sustenta la predicación análoga de los nombres

11 Cf. In I Sent., d. 4, q. 1, a. 1; ST I, q. 13, a. 1, ad 2; De pot., q. 8, a. 2, ad 7. 
divinos sólo nos permite aproximarnos a las perfecciones divinas, su esencia siempre permanece incomprendida. Ciertamente, como hemos venido argumentando, la estructura básica de la predicación análoga está presente en todos casos, pero la trascendencia divina hace que el caso de los nombres divinos no pueda subsumirse por completo a ninguna de las tipologías tradiciones de analogía (tipologías que de por sí nunca fueron del todo consistentes). Esto explicaría el hecho de que Tomás vacila en su tratamiento de la analogía de los nombres divinos, ensayando una y otra tipología, unas veces equiparando el caso de los nombres divinos con el caso de los términos 'ente' y 'sano', otras veces distinguiéndolos. Más allá de estas vacilaciones, sin embargo, a lo largo de su obra, Tomás apela tanto a la noción de analogía como al principio de causalidad ejemplar y eficiente en su tratamiento del problema de los nombres divinos (Porro, 2018, p. 77). La teoría de la analogía, junto con la noción de causalidad formal y eficiente, ofrece a Tomás la posibilidad de mantener un fino equilibrio entre la absoluta trascendencia de Dios y nuestra habilidad de predicar algo positivo de Dios, de tal manera que se pueda garantizar una ciencia sobre Dios.

\section{REFERENCIAS}

Ashworth, E. J. (1992). Analogy and Equivocation in Thirteenth Century Logic: Aquinas in Context. Mediaeval Studies, 54, 94-135.

Ashworth, E. J. (1996). Analogy, Univocation, and Equivocation in Some Early Fourteenth-Century Authors. En J. Marenbon (Ed.), Aristotle in Britain during the Middle Ages (pp. 233-247). Brepols.
Ashworth, E. J. (2008). Les théories de l'analogie du XIle au XVIe siècle. Vrin.

Buersmeyer, K. (1987). Aquinas on the Modi Significandi. Modern Schoolman, 64 (2), 73-95.

De Rijk, L. M. (Ed.) (1972). Peter of Spain. Tractatus, called afterwards Summule Logicales. Van Gorcum.

Jordan, M. (1980). Modes of Discourse in Aquinas' Metaphysics. New Scholasticism 54, 80-98.

Lohr, C. H. (1965). Logica Algazelis: Introduction and Critical Text. Traditio, 21, 223-290.

Lonfat, J. (2004). Archéologie de la notion d'analogie d'Aristote à Saint Thomas d'Aquin. Archives d'histoire doctrinale et littéraire du Moyen Âge, 71, 35-107.

Porro, P. (2018). Tomás de Aquino. Un perfil histórico-filosófico. UCSS.

Rosier, I. (1995). Res significata et modus significandi: Les implications d'une distinction médiévale. En S. Ebbesen (Ed.), Sprachtheorien in Spätantike und Mittelalter (pp. 135-168). Gunter Narr Verlag.

Fecha de recepción: 13-11-2020 Fecha de aceptación: 29-11-2020 